\title{
ANESTHESIA PREOPERATIVE EVALUATION CLINIC (APEC) RESTRUCTURING PLAN FOR IMPROVING THE QUALITY OF SURGICAL PATIENT FLOW
}

\author{
Carolina Alboim ${ }^{1}$, Alice Becker Teixeira1, Luciana Eltz Soares², \\ Vanda Regina Machado ${ }^{3}$, Luciana Cadore Stefani ${ }^{4,5}$
}

Clin Biomed Res. 2018;38(3):258-264

1 Serviço de Anestesia e Medicina Perioperatória (SAMPE), Hospital de Clínicas de Porto Alegre (HCPA). Porto Alegre, RS, Brasil.

2 Universidade Federal do Rio Grande do Sul (UFRGS). Porto Alegre, RS, Brasil.

3 Coordenadoria Administrativa, Serviço de Atenção Cirúrgica, Serviço de Anestesia e Medicina Perioperatória (SAMPE), Hospital de Clínicas de Porto Alegre. Porto Alegre, RS, Brasil.

4 Programa de Pós-graduaçăo em Medicina, Faculdade de Medicina, Universidade Federal do Rio Grande do Sul. Porto Alegre, RS, Brasil.

5 Serviço de Cirurgia, Hospital de Clínicas de Porto Alegre. Porto Alegre, RS, Brasil.

Corresponding author:

Carolina Alboim

calboim@hcpa.edu.br

Serviço de Anestesia e Medicina Perioperatória (SAMPE), Hospital de Clínicas de Porto Alegre (HCPA) Rua Ramiro Barcelos, 2350, $13^{\circ}$ andar. 90035-903, Porto Alegre, RS, Brasil.

\section{ABSTRACT}

Introduction: Anesthesia preoperative evaluation clinics (APECs) are useful for high-risk surgical patient care, as they improve perioperative outcomes and optimize patient care flow. At Hospital de Clínicas de Porto Alegre, APEC assesses $20 \%$ of all patients undergoing surgery, showing the importance of implementing strategies to improve outpatient clinic efficiency by prioritizing high-risk patients.

Methods: Using a specific quality management tool for process improvement (PDCA, which stands for Plan/Do/Check/Act), new protocols were developed for patient referral and preoperative evaluation. Clinical staff was trained in the new routines, and an electronic screening system for patient referral was adopted. Data on patient profiles, referring surgical specialties, type of surgery, and waiting times for appointment and surgical procedure were compared before and after the intervention.

Results: APEC performed 1,286 appointments between 2013 and 2016, including 733 pre-intervention and 553 post-intervention. There was a significant decrease in the appointments for patients undergoing minor surgery and an increase in the appointments for those undergoing major surgery. The waiting time between referral to APEC and first APEC appointment reduced from 46 to 16 days. In addition, there was an increase in vascular, orthopedic, and urology referrals, as well as a reduction in ophthalmology and general surgery referrals.

Conclusions: The PDCA method assisted with conceiving, executing, and monitoring the implemented strategies for changing the profile of patients evaluated at APEC. The major challenge is to measure the long-term impact of this intervention and expand care through specific strategies for surgical specialties and procedures.

Keywords: Preanesthesia evaluation; preoperative evaluation; preoperative stratification; risk stratification

Overall perioperative risk depends on the interaction between several factors relating to anesthesia, patient's clinical conditions, and surgery. Preoperative evaluation is included in a hospital's safety and quality policy and aims to identify and stratify medical-surgical risk in order to improve perioperative outcomes and optimize patient care flow ${ }^{1,2}$. In addition, previous knowledge of risks associated with the combination of comorbidities and surgical procedure allows reducing care fragmentation and creating integrated multidisciplinary care strategies, with emphasis on standardizing processes and adopting evidence-based measures ${ }^{2,3}$.

Preanesthesia or preoperative evaluation clinics with multidisciplinary teams focused on the perioperative period have grown in recent years ${ }^{1}$. Although the effect of preoperative evaluation on reduced morbidity and mortality is unclear in the literature ${ }^{4-7}$, some studies have demonstrated benefits such as increased surgical volume, reduced time between surgical indication and surgery, fewer surgery cancellations, and improved patient education regarding perioperative care. The greatest impact has been observed in high-risk patients and major surgeries ${ }^{1,8,9}$. 
The benefits of outpatient preoperative evaluation include optimized flow at surgical units. A cohort study of over 5,000 patients in Chicago demonstrated that surgical waiting time was longer when no preoperative evaluation was performed. The number of cancellations was 2 times higher in this group compared with patients who underwent the assessment, strongly suggesting that preoperative evaluation plays an important role in reducing cancellation rates and surgery delays ${ }^{10}$. Another recent study showed benefit in reducing operating room entry delays and turnover times, even for low-risk patients ${ }^{11}$. However, there is still no clear recommendation supporting the process of outpatient preoperative evaluation for all patients regardless of the risk ${ }^{12,13}$.

In 1999, the Anesthesia and Perioperative Medicine Service (SAMPE) at Hospital de Clínicas de Porto Alegre (HCPA), in a multidisciplinary effort, initiated its anesthesia preoperative evaluation clinic (APEC). APEC structure and functions are similar to those described by Vetter et al. within a new model of care proposed by the American Society of Anesthesiologists (ASA), named Perioperative Surgical Home (Table 1).

Before mid-2015, there was no formal restriction to which surgical specialties could refer patients to APEC; rather, there were guidelines on whom should receive priority care, i.e., patients with multiple comorbidities, regardless of the referring specialty. Referred patients were scheduled according to available appointment times. However, several problems were identified as a result of this scheduling system, as the large HCPA surgical volume (approximately 19,000 surgeries/year) is incompatible with APEC appointment capacity (approximately 3,000 appointments/year). A2013 report showed that only a small number of appointments concerned major surgeries and great disparity was observed between the referring specialties. In addition, waiting time between referral to APEC and first APEC appointment was greater than 70 days, thus preventing a prompt evaluation of time-sensitive surgeries and high-risk patients ${ }^{14}$.

The present study describes the strategies adopted by SAMPE to restructure its APEC and examines the results obtained 6 months after the implementation of an improvement plan based on the use of a quality management tool known by the abbreviation PDCA (Plan/Do/Check/Act). The purpose was to prioritize the care of patients of higher medical risk and/or undergoing major procedures, as well as to reduce the time between referral and appointment.

\section{METHODS}

This retrospective observational study included patients having their first APA appointment. Data were collected from the HCPA Management Information System regarding two periods in APEC history, i.e., pre-intervention (January 2013 to March 2014) versus post-intervention (June 2015 to June 2016).

Medical records were reviewed by two trained researchers. Patients who failed to attend the appointment and those who had their surgery canceled were excluded. The following variables were collected: medical record number, ASA physical status classification included in the surgical description, date of referral to APEC, date of APEC appointment, date of surgery, referring medical specialty, and type of surgical procedure. Surgical procedures were categorized according to their severity into minor, moderate, and major. This classification was based

Table 1: Preoperative steps of the Perioperative Surgical Home integrated program.

Perform a comprehensive evaluation of the patient at the specialized preoperative clinic

Identify, communicate, and/or minimize specific comorbidities of the patient, and record risk of surgery and anesthesia

Apply established clinical protocols for preoperative diagnostic tests and for clinical optimization

Implement an individualized perioperative care plan including additional preoperative medications and maintenance of chronic medications

Provide patient education regarding surgical procedure with the purpose of reducing anxiety, increasing patient engagement, and improving postoperative recovery

Obtain informed consent

Collect a detailed clinical and surgical history at the clinic

Reduce delays and cancellations through clinical optimization prior to the day of surgery, and perform protocolguided diagnostic tests

Use electronic medical record to communicate appointment findings and suggest approaches to intra- and postoperative anesthetic care

Source: Adapted from Vetter TR, et al Preoperative elements of the Perioperative Surgical Home model at the University of Alabama at Birmingham. 
on the study of Glance et al. and adapted to the list of procedures provided in the HCPA information system ${ }^{15}$. A detailed description of the present surgical procedure classification is found in a related article about the development of a postoperative mortality risk model (named SAMPE model) ${ }^{16}$.

The PDCA quality management tool was used for defining APEC efficiency improvement strategies as described in Figure 1. This method is largely employed by companies seeking to improve their management level through efficient control of internal and external processes and activities, providing standardized information and reducing errors in decision-making ${ }^{17-19}$. Importantly, the implemented PDCA cycle should become a standard within the company in order to promote constant improvement. In Figure 2, a flowchart shows the steps in implementing APEC care improvement processes using the PDCA method.

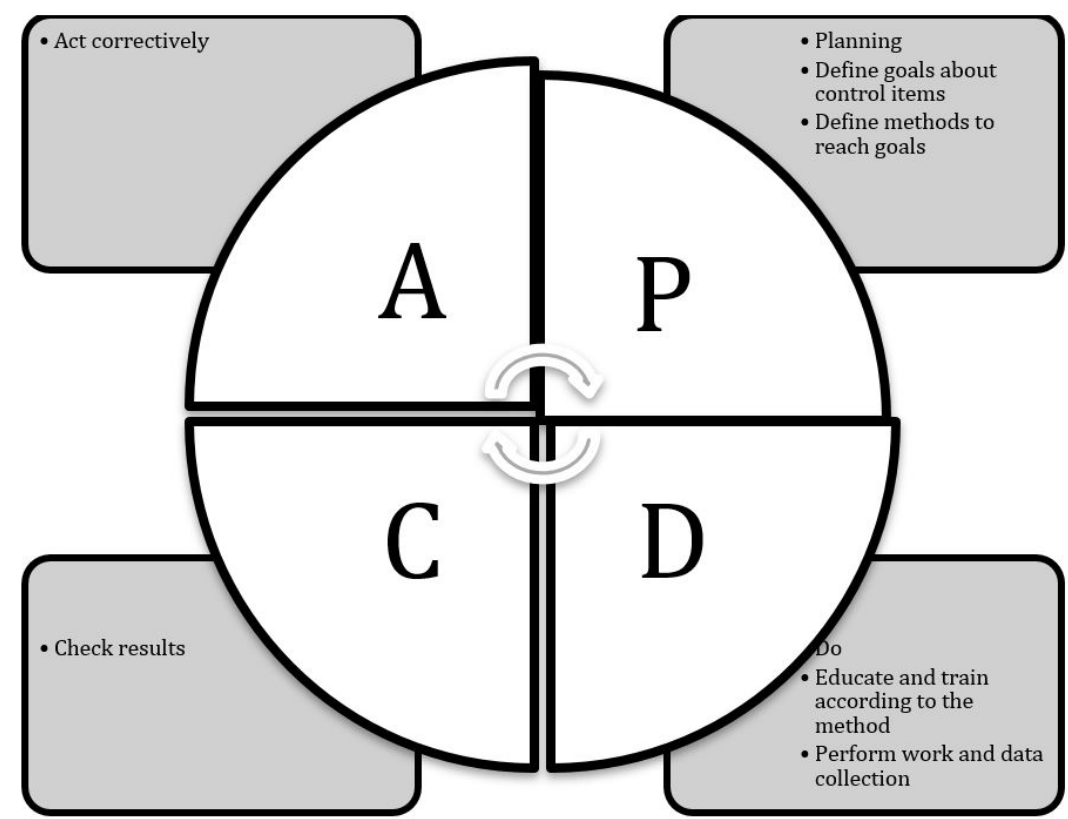

Figure 1: PDCA tool used for implementation of care quality improvement.

\begin{tabular}{|l|l|}
\hline P- Planning & $\begin{array}{l}\text { The analysis of the profile of patients treated between } 2013 \text { and } 2014 \text { revealed a } \\
\text { predominance of low risk patients, long waiting time, and proportion of patients } \\
\text { referred by specialty. }\end{array}$ \\
\hline
\end{tabular}

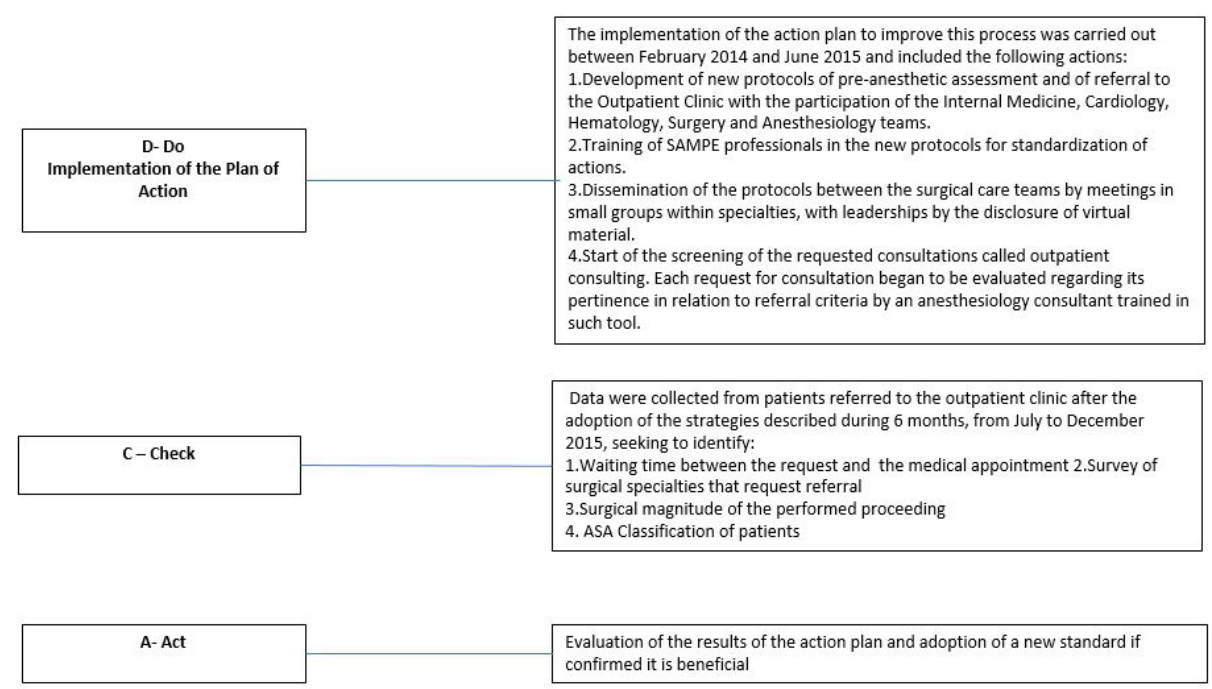

Figure 2: Flow of the Restructuring of the Pre-Anesthetic Evaluation Outpatient Clinic (APEC) using the PDCA strategy. 
During the implementation of this action plan, the development and dissemination of a care protocol was crucial for assisting surgical teams in deciding which patients should be referred to APEC. Furthermore, an appointment screening system via consultant anesthesiologist was used for selecting appointments and returning to surgeons those patients considered low-risk or with no factors requiring preoperative evaluation or compensation.

\section{Statistical Analysis}

The variables were compared between 2013 and 2016 -- before and after the implementation of the APEC restructuring plan. The following measures were calculated in the post-restructuring outcome analysis: mean and median waiting times between referral to APEC and first APEC appointment (T1), between APEC appointment and surgery (T2), and between referral to APEC and surgery (T3). The Mann-Whitney test was used for nonparametric quantitative variables. The chi-square test was used for calculating differences between ASA classification and surgical severity before and after the intervention, as well as the specialties requesting APEC evaluation.

The analysis was performed using SPSS for Windows, version 22. A $p<0.05$ was considered statistically significant.

\section{RESULTS}

Of a total 1,286 first APEC appointments scheduled for the period between 2013 and 2016, 733 pre-intervention appointments (2013-2014) and 553 post-intervention appointments (2015-2016) were included in the analysis, as shown in figure 3.

There was a significant decrease in T1, T2, and T3, as demonstrated in Table 2. In 2013, 55 (7.5\%) patients waited for so long that their surgery was performed before their APEC appointment. There was a decrease in wasted scheduled appointments after the intervention, with a drop from $7.5 \%$ to $2 \%$ in the number of patients whose surgery was performed before the scheduled APEC appointment.

No significant difference was observed in ASA classification of patients evaluated at APEC, but the number of ASA 1 patients decreased after the intervention ( $10 \%$ vs. $6.8 \% ; p=0.106)$. Regarding surgical severity, there was a significant reduction in the appointments of patients undergoing minor procedures $(63.2 \%$ vs. $54.1 \% ; p<0.001)$, as well as an increase in the number of patients undergoing major procedures $(13.9 \%$ vs. $23 \% ; p<0.001)$. There was no significant change in the number of patients undergoing moderate procedures (Table 3 ).

Regarding referring surgical specialties, there was a significant decrease in referrals from general

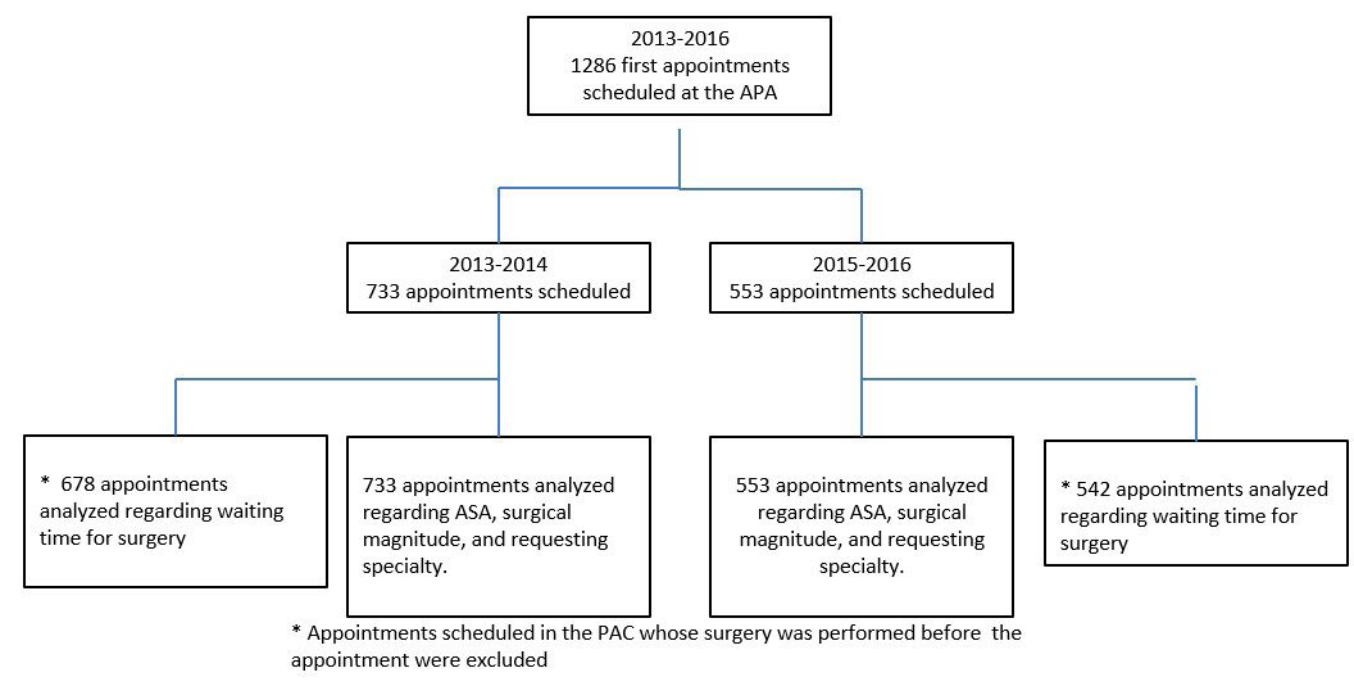

Figure 3: Flowchart illustrating the inclusion of the first appointments in the two stages evaluated: before (2013-2014) and after (2015-2016) the intervention.

Table 2: Comparison of time (days) between referral to anesthesia preoperative evaluation clinic (APEC) and first APEC appointment (T1), between APEC appointment and surgery (T2), and between referral to APEC and surgery (T3).

\begin{tabular}{cccrl}
\hline APEC & $\mathbf{2 0 1 3 / 2 0 1 4}$ & $\mathbf{2 0 1 5 / 2 0 1 6}$ & $\mathbf{F}$ & $\mathbf{P}$ \\
appointment & $\mathbf{n}=\mathbf{6 7 8}$ & $\mathbf{n}=\mathbf{5 4 2}$ & 0.00 & $<0.001$ \\
T1 & $46.1 \pm 18(47)$ & $16.8 \pm 14.9(13)$ & 11.84 & $<0.001$ \\
T2 & $103.1 \pm 76.3(83)$ & $84.1 \pm 68.4(68.3)$ & 16.18 & $<0.001$ \\
T3 & $149.8 \pm 80.9(131)$ & $101.4 \pm 69.1(84.6)$ & & \\
\hline
\end{tabular}


surgery $(16.8 \%$ vs. $9.6 \% ; p<0.001)$, ophthalmology $(14.1 \%$ vs. $9.6 \% ; p=0.01)$, and pediatric surgery $(0.7 \% \times 0.0 \% ; p=0.03)$ teams. The number of appointments for patients of the following specialties increased: orthopedics (6.7\% vs. $10.5 \%$; $p<0.001)$, gynecology $(12.7 \%$ vs. $17.5 \% ; p=0.01)$, urology $(5.9 \%$ vs. $11.6 \% ; p<0.001)$, and vascular surgery $(0.3 \%$ vs. $1.6 \% ; p=0.04)$ (Table 4$)$.

\section{DISCUSSION}

With the implementation of the APEC restructuring plan using the PDCA method, a significant change was observed in the profile of the population evaluated at the clinic, with a growing number of patients undergoing major surgeries versus minor surgeries. There was a $65 \%$ increase in the appointments for the former population, regarded as higher perioperative risk. Another significant finding was a reduction in mean waiting time for APEC appointment, which dropped from 46 to 16 days. The waiting time between referral to APEC and surgery also reduced. This efficiency gain resulted from the adoption of a screening system via consultant anesthesiologist, who, using predefined criteria, did not allow scheduling appointments for patients with no comorbidities and/or undergoing minor surgeries. This measure had a sustained impact, as the 2012-2017 referral waiting time indicator (Figure 4) demonstrated an important reduction in referral waiting time after 2015. The creation and implementation of this tool as a SAMPE care indicator was relevant considering the cyclical nature of the PDCA method, allowing continuous result checking.

The results show a direct impact on the improvement of surgical patient flow, as surgical teams were not referring their patients due to long waiting times, especially for time-sensitive surgeries, in which time affects outcomes, such as surgical oncology. This change makes sense in a context where the APEC has limited capacity when faced with the hospital's large surgical volume. Additionally, there has been a progressive increase in the number of outpatient surgeries of low complexity or using less invasive techniques. The cost-effectiveness of outpatient preoperative or specialized evaluations is questionable in such cases ${ }^{20}$.

The criteria used by surgical specialties to determine which patients should undergo preoperative evaluation vary according to each context and country.

Table 3: Comparison between surgical magnitudes before and after the implementation of the restructuring plan.

\begin{tabular}{|c|c|c|c|}
\hline Magnitude & $\begin{array}{c}\text { Before } \\
2013 / 2014(n=733) \\
n(\%)\end{array}$ & $\begin{array}{c}\text { After } \\
2015 / 2016(n=553) \\
n(\%)\end{array}$ & $\mathbf{P}$ \\
\hline Minor & $463(63.2)$ & $299(54.1)$ & $<0.001$ \\
\hline Moderate & $168(22.9)$ & $127(23)$ & 0.92 \\
\hline
\end{tabular}

Table 4: Comparison between surgical specialties that requested anesthesia preoperative evaluation clinic (APEC) appointments before and after the intervention.

\begin{tabular}{lccc}
\hline \multicolumn{1}{c}{ Referring specialties } & $\mathbf{2 0 1 3 / 2 0 1 4}$ & $\mathbf{2 0 1 5 / 2 0 1 6}$ \\
& $\mathbf{n = 7 3 3}$ & $\mathbf{n}=\mathbf{5 5 3}$ & $\mathbf{n}(\mathbf{\%})$ \\
\hline General surgery & $\mathbf{n}(\mathbf{\%})$ & $53(9.6)$ & 0.00 \\
Orthopedics & $123(16.8)$ & $58(10.5)$ & 0.00 \\
Gynecology & $49(6.7)$ & $97(17.5)$ & 0.01 \\
Urology & $93(12.7)$ & $64(11.6)$ & 0.00 \\
Ophthalmology & $43(5.9)$ & $53(9.6)$ & 0.01 \\
Abdominal surgery & $103(14.1)$ & $95(17.2)$ & 0.13 \\
Head and neck surgery & $149(20.3)$ & $31(5.6)$ & 0.23 \\
Proctology & $55(7.5)$ & $29(5.2)$ & 0.23 \\
Plastic surgery & $28(3.8)$ & $12(2.2)$ & 0.08 \\
Pediatric surgery & $8(1.1)$ & 0 & 0.03 \\
Breast surgery & $5(0.7)$ & $19(3.4)$ & 0.92 \\
Thoracic surgery & $24(3.3)$ & $3(0.5)$ & 0.42 \\
Oral and maxillofacial surgery & $2(0.3)$ & $23(4.2)$ & 0.1 \\
Surgical oncology & $44(6.0)$ & $3(0.5)$ & 0.76 \\
Vascular surgery & $5(0.7)$ & $9(1.6)$ & 0.04 \\
Neurosurgery & $2(0.3)$ & $3(0.5)$ & 0.27 \\
\hline
\end{tabular}




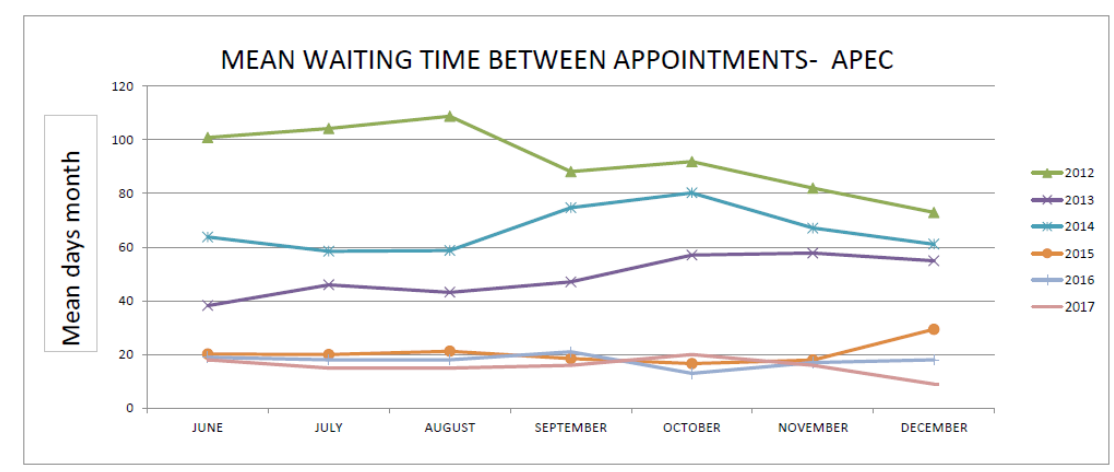

Figure 4: Waiting time for surgical referral appointment at the anesthesia preoperative evaluation clinic (APEC).

An observational study of 556,637 patients has found that ophthalmology referrals were primarily driven by non-medical factors, such as local practices and geographic variation ${ }^{21}$. In the present study, the specialties requesting the highest number of APEC evaluations before the intervention were general surgery, digestive surgery, and ophthalmology. With the restructuring plan, urologic, orthopedic, and vascular patients -- usually older adults with several comorbidities -- increased from $12.8 \%$ in $2013 / 2014$ to $23.6 \%$ in $2015 / 2016$. The referral rate of patients undergoing thoracic and oncologic surgery remained low, despite being patients of higher surgical risk and with more comorbidities, indicating that there is room for improving the care strategies in place for these specialties. Some specialties such as oral and maxillofacial surgery and pediatric surgery reduced the number of referrals after the implementation of the action plan. The explanation is that most patients are young and ASA 1. Similarly, there was a significant reduction in APEC appointments for ophthalmic patients. A recent study of patients undergoing cataract surgery found no differences in major perioperative complications between those who underwent preoperative evaluation and those who did not, suggesting that this assessment has no effect on reducing adverse events in this population ${ }^{22}$.

The adoption of an appointment screening system via consultant anesthesiologist was a key measure for improving surgical patient flow. This system was useful for guiding the teams with regard to referral criteria established in the new protocol, thus expediting the process. However, this measure did not reduce the number of ASA 1 or 2 patients evaluated at the clinic. Although these patients could a priori undergo surgery without preoperative evaluation because of their lower surgical risk, other factors that do not interfere with ASA classification are used as referral criteria, such as moderate and major surgeries, difficult airway, rheumatic diseases, history of allergies, or previous anesthetic complications. This is probably why no difference in ASA classification was found in the present study.

The present study has some limitations. First, data are retrospective and depend on the quality of medical records and surgical descriptions. Second, the ASA classification, which was used for identifying individual medical severity, is somewhat subjective. To reduce bias, SAMPE recommends using the updated ASA classification, which shows examples of clinical situations for each category ${ }^{23}$. At HCPA, several factors contribute to surgery cancellations, including lack of evaluation or clinical decompensation, together with scheduling failures and lack of equipment or material. The effect of preoperative evaluation on surgery cancellation rate could not be determined.

This study demonstrated that the adoption of a process improvement tool such as PDCA assisted with conceiving, executing, and monitoring the implemented strategies. The greatest challenge is to measure the long-term impact of the observed change in the profile of the APEC population and its effect on clinical and economic outcomes at HCPA. Moreover, a future agenda should include an expansion with specific multidisciplinary care strategies for surgical specialties or procedures, focused on perioperative care, which covers from surgery preparation to late rehabilitation aimed at functional recovery after surgical trauma. The PDCA tool will be extremely useful once more in the development of this future expansion.

\section{Conflitos de Interesse}

The authors declare no conflicts of interest. 


\section{REFERENCES}

1. Lee A, Kerridge RK, Chui PT, Chiu $\mathrm{CH}$, Gin T. Perioperative Systems as a quality model of perioperative medicine and surgical care. Health Policy. 2011;102(2-3):214-22. http://dx.doi. org/10.1016/j.healthpol.2011.05.009. PMid:21680044.

2. Cohn SL, Mauck KF, Dutta S, Jaffer A Smetana GW. Update in perioperative medicine 2013. Hosp Pract (1995). 2013;41(4):15-23. PMid:24145585.

3. Vetter TR, Boudreaux AM, Jones KA, Hunter JM JR, Pittet JF. The perioperative surgical home: how anesthesiology can collaboratively achieve and leverage the triple aim in health care. Anesth Analg. 2014;118(5):1131-6. http://dx.doi. org/10.1213/ANE.0000000000000228. PMid:24781579.

4. Cantlay KL, Baker S, Parry A, Danjoux $G$. The impact of a consultant anaesthetist led pre-operative assessment clinic on patients undergoing major vascular surgery. Anaesthesia. 2006;61(3):234-9. http://dx.doi.org/10.1111/j.13652044.2005.04514.x. PMid:16480347.

5. Wijeysundera DN, Austin PC, Beattie WS, Hux JE, Laupacis A. Outcomes and processes of care related to preoperative medical consultation. Arch Intern Med. 2010;170(15):1365-74. http://dx.doi. org/10.1001/archinternmed.2010.204. PMid:20696963.

6. Auerbach AD, Wachter RM, Cheng $H Q$, Maselli J, McDermott M, Vittinghoff $\mathrm{E}$, et al. Comanagement of surgical patients between neurosurgeons and hospitalists. Arch Intern Med. 2010;170(22):200410. http://dx.doi.org/10.1001/ archinternmed.2010.432. PMid:21149758.

7. Hofer J, Chung E, Sweitzer BJ. Preanesthesia evaluation for ambulatory surgery: do we make a difference? Curr Opin Anaesthesiol. 2013;26(6):66976. http://dx.doi.org/10.1097/ ACO.0000000000000006. PMid:24184883.

8. Wijeysundera DN. Preoperative consultations by anesthesiologists. Curr Opin Anaesthesiol. 2011;24(3):326-
30. http://dx.doi.org/10.1097/ ACO.0b013e328345d844. PMid:21451401.

9. Biccard BM, Devereaux PJ, Rodseth RN. Cardiac biomarkers in the prediction of risk in the non-cardiac surgery setting. Anaesthesia. 2014;69(5):484-93. http://dx.doi.org/10.1111/anae.12635. PMid:24738805.

10. Ferschl MB, Tung A, Sweitzer B, Huo $D$, Glick DB. Preoperative clinic visits reduce operating room, cancellations and delays. Anesthesiology. 2005;103(4):855-9. http://dx.doi. org/10.1097/00000542-20051000000025. PMid:16192779.

11. Epstein RH, Dexter F, Schwenk ES, Witkowski TA. Bypass of anesthesiologist - diretct preoperative evaluation clinic results in greater first - case tardiness and turnover times. J Clin Anesth. 2017;41:1129. http://dx.doi.org/10.1016/j. jclinane.2017.04.009. PMid:28438362.

12. Auerbach $A D$, Rasic MA, Sehgal $\mathrm{N}$, Ide B, Stone B, Maselli J. Opportunity missed: Medical consutation, resource use, and quality of care of patients undergoing major surgery. Arch Intern Med. 2007;167(21):2338-44. http://dx.doi. org/10.1001/archinte.167.21.2338. PMid:18039993.

13. Wijeysundera DN, Austin PC, Beattie WS, Hux JE, Laupacis A. Outcomes and processes of care related to preoperative medical consutation. Arch Intern Med. 2010;170(15):1365-74. http://dx.doi. org/10.1001/archinternmed.2010.204. PMid:20696963.

14. Cadore L. Ambulatório de avaliação pré-anestésica do Hospital de Clínicas de Porto Alegre (APA): Análise das especialidades solicitantes e risco cirúrgico entre 1377 consultas consecutivas. Clin Biomed Res. 2014;34:110.

15. Glance LG, Lustik SJ, Hannan EL, Osler TM, Mukamel DB, Qian F, et al. The surgical mortality probability model: Derivation and validation of a simple risk prediction rule for noncardiac surgery. Ann Surg 2012;255(4):696-702. http://dx.doi. org/10.1097/SLA.0b013e31824b45af. PMid:22418007.
16. Stefani LC, Gutierrez CS, Castro SMJ, Zimmer RL, Diehl FP, Meyer LE, et al. Derivation and validation of a preoperative risk model for postoperative mortality (SAMPE model): An approach to care stratification. PLoS One. 2017;12(10):1-14.

17. Carter J. Small-scale study using the PDCA cycle. In: Gift R, Kinney C, Shortell S, editors. Today's Management Methods: A guide for the health care executive. New York: Wiley, John \& Sons, Inc.; 1996. P. 209-22.

18. Mori K, Takebayashi T. The introduction of an occupational health management system for solving issues in occupational health activities in Japan. Ind Health. 2002;40:167-17.

19. Williams HF, Fallone S. CQI in the acute care setting: An opportunity to influence acute care practice. Nephrol Nurs J. 2008;35(5):515-22. PMid:18856084.

20. Keay L, Lindsley K, Tielsch J, Katz $\mathrm{J}$, Schein O. Routine preoperative medical testing for cataract surgery. Cochrane Database Syst Rev. 2012;3(3):CD007293. PMid:22419323.

21. Thilen SR, Treggiari MM, Lange JM, Lowy E, Weaver EM, Wijeysundera DN. Preoperative consultations for medicare patients undergoing cataract surgery. JAMA Intern Med. 2014;174(3):3808. http://dx.doi.org/10.1001/ jamainternmed.2013.13426. PMid:24366269.

22. Alboim C, Kliemann RB, Soares LE, Ferreira MM, Polanczyk CA, Biolo A The impact of preoperative evaluation on perioperative events in patients undergoing cataract surgery: a cohort study. Eye (Lond). 2016;30(12):161422. http://dx.doi.org/10.1038/ eye.2016.203. PMid:27636228.

23. American Society of Anesthesiologists (ASA). Physical Status Classification System. Last approved by the ASA House of Delegates on October 15, 2014. [cited 2018 April 15]. Available from: http://www.asahq.org/resources/ clinical-information/asaphysical-statusclassification-system 